\title{
Fast Field-Cycling Magnetic Resonance Imaging
}

David J. Lurie ${ }^{1}$, Silvio Aime ${ }^{2}$, Simona Baroni ${ }^{3}$, Nuala A. Booth ${ }^{4}$, Lionel M. Broche ${ }^{1}$, Chang-Hoon Choi ${ }^{1}$, Gareth R. Davies ${ }^{1}$, Saadiya Ismail ${ }^{1,4}$, Dara Ó hÓgáin ${ }^{1}$, Kerrin J. Pine

\footnotetext{
${ }^{1}$ Aberdeen Biomedical Imaging Centre, University of Aberdeen, Foresterhill, Aberdeen AB25 2ZD, Scotland, UK

${ }^{2}$ Department of Chemistry 'IFM', University of Torino, Italy

${ }^{3}$ Invento S.r.1., Via Nizza 52, I-10126, Torino, Italy

${ }^{4}$ Institute of Medical Sciences, University of Aberdeen, Foresterhill, Aberdeen AB25 2ZD, Scotland, UK
}

Email: d.lurie@abdn.ac.uk

Phone: +44 1224554061

Fax: +44 1224552514

Keywords: NMR, field-cycling, magnetic resonance imaging, quadrupole dips 
Abstract

Magnetic resonance imaging (MRI) and fast field-cycling (FFC) NMR are both well-developed methods. The combination of these techniques, namely fast field-cycling magnetic resonance imaging (FFC-MRI) is much less well-known. Nevertheless, FFC-MRI has a number of significant applications and advantages over conventional techniques, and is being pursued in a number of laboratories. This article reviews the progress in FFC-MRI over the last two decades, particularly in the areas of Earth's field and pre-polarised MRI, as well as free radical imaging using field-cycling Overhauser MRI. Different approaches to magnet design for FFC-MRI are also described. The paper then goes on to discuss recent techniques and applications of FFC-MRI, including protein measurement via quadrupolar cross-relaxation, contrast agent studies, localised relaxometry and FFCMRI with magnetisation-transfer contrast. 


\section{Introduction}

Magnetic field-cycling has been in use for several decades, and is now a routine method to measure the nuclear magnetic resonance (NMR) relaxation behaviour as a function of resonant frequency (or, equivalently, magnetic field strength). A plot of a sample's $T_{1}$ relaxation time as a function of Larmor frequency is known as a $T_{1}$-dispersion plot. Currently-available commercial field-cycling relaxometers allow these measurements to be made from virtually zero magnetic field up to approximately 1 tesla. Magnetic resonance imaging (MRI) - at least, the conventional variety - is even more ubiquitous, being found in a large percentage of hospitals around the world, as well as in research centres and laboratories. By contrast, the marriage of these techniques, namely field-cycling magnetic resonance imaging (FC-MRI), is still relatively uncommon, being so far limited to a handful of research laboratories. Nevertheless, FC-MRI offers several intriguing possibilities and a number of novel applications. This article will explore the history, instrumentation, methods and applications of FC-MRI.

\section{Magnetic Field-Cycling NMR}

A number of excellent reviews have been written, describing the techniques and applications of fieldcycling NMR [1-4]. The basic premise of field-cycling magnetic resonance is that the magnetic field is switched between levels during the pulse sequence, as shown in Figure 1. In the general case, as depicted there, the pulse sequence comprises three periods, namely Polarisation, Evolution and Detection, with magnetic fields being applied at different values $\left(B_{0}{ }^{\mathrm{P}}, B_{0}{ }^{\mathrm{E}}\right.$ and $\left.B_{0}{ }^{\mathrm{D}}\right)$ during each period. The high magnetic field applied during the Polarisation period serves to increase the Boltzmann nuclear magnetisation. As its name suggests, the spin system evolves during the Evolution period, the duration of which is typically altered in order to deduce the sample's relaxation behaviour at the chosen $B_{0}{ }^{\mathrm{E}}$ value. A crucial aspect of field-cycling magnetic resonance is that during the measurement of dispersion data, signal detection is always carried out at the same magnetic field, $B_{0}{ }^{\mathrm{D}}$, at the same resonant frequency (equal to $\gamma B_{0}{ }^{\mathrm{D}}$ where $\gamma$ is the gyromagnetic ratio of the nuclear spin system under study); this means that the behaviour of the spins can be studied at a wide range of 
magnetic field strengths, without the need to re-tune or adjust the instrument's radiofrequency (RF) coils. A further advantage is that the magnetic field resolution of the dispersion plot can be as fine or as coarse as is required by the experimenter (within the constraints of the instrument's magnetic field controller). In most field-cycling experiments the switching time between fields is significantly shorter than the sample's spin-lattice relaxation time, $T_{1}$. In that case, the method is known as "Fast Field-Cycling" (FFC).

\section{Field-cycling with Magnetic Resonance Imaging}

A number of diverse uses of field-cycling in conjunction with MRI have been reported, which will now be discussed.

\subsection{Pre-polarised MRI}

"Pre-polarisation" describes the procedure of applying an increased magnetic field in order to boost a sample's nuclear magnetisation, prior to signal detection at a lower field. The first example of prepolarised magnetic resonance was published in 1954 by Packard and Varian, who used the method in order to measure a sample's free induction decay signal in the Earth's magnetic field [5]. The prepolarisation magnetic field of $10 \mathrm{mT}$ was applied, then switched off non-adiabatically, leaving the magnetisation precessing about the Earth's field. More recently, pre-polarisation has been used together with MRI in order to image objects in the Earth's magnetic field, as described in section 3.2 of this paper.

The term "pre-polarised MRI" (PMRI) has come to be associated with a method where prepolarisation is used to boost the magnetisation prior to detection (along with the application of magnetic field gradients) at a lower magnetic field, with the pre-polarising field and the readout field both being generated by resistive magnet coils. Macovski and Conolly at Stanford University originally suggested this approach, with the intention of building a low-cost MRI scanner that would nevertheless possess high sensitivity, by virtue of the (relatively) high-field pre-polarisation [6]. A typical pulse sequence for PMRI is shown in Figure 2. The Stanford group's implementation of PMRI 
includes a pre-polarisation coil capable of generating $0.42 \mathrm{~T}$, located inside a 6-coil readout magnet operating at up to $60 \mathrm{mT}$ (2.5 MHz proton Larmor frequency) [7]. It has been shown to be capable of generating good quality images, which are relatively immune from artefacts around metal orthopaedic implants, due to the low readout field [8]. A design for a pre-polarised field-cycling MRI system for small animal imaging was also published by Gilbert et al. [9], and the same group at the University of Western Ontario also showed that field-cycling could be used to construct a combined Positron Emission Tomography (PET) and MRI scanner [10]. The advantage of field-cycling here is that the magnetic field can be switched off at certain times during the pulse sequence to allow detection of the positron-emitting radioisotope by scintillators coupled to conventional photomultiplier tube detectors, then switched on again for NMR signal detection. Without field-cycling, the efficiency of the PET scanner would be significantly compromised.

\subsection{Earth's magnetic field FC-MRI}

An extreme case of pre-polarised MRI is its use in Earth's field imaging. The Earth's magnetic field is very weak ( $\sim 50 \mu \mathrm{T}$, depending on location), but potentially very homogeneous, offering the potential for NMR and MRI on large objects. Since the Boltzmann polarisation is extremely feeble at this field strength, pre-polarisation using field-cycling can be used to boost the sample's magnetisation, prior to signal detection in the Earth's field at a Larmor frequency of $\sim 2 \mathrm{kHz}$ (for protons). Such a scheme was demonstrated by Stepisnik et al. in 1990 [11]. They used a solenoid to generate a polarisation magnetic field of $30 \mathrm{mT}$, oriented perpendicular to the Earth's field. Its field is switched off adiabatically so that the nuclear magnetisation is realigned with the Earth's field, and precesses about that direction following the application of a $90^{\circ}$ pulse. As a result, the same coil could be used to detect the NMR signals generated by the precessing magnetisation. The authors were able to generate images of water-filled tubes and of various fruits, with a slice thickness of $\sim 1 \mathrm{~cm}$ and in-plane resolution of $\sim 2 \mathrm{~mm}$. More recently, the same group has published details of an improved Earth's field imaging system, this time using pre-polarisation at $60 \mathrm{mT}$ [12]. Other workers have taken a similar approach, using pre-polarisation prior to signal detection in the Earth's field [13] or even at zero field [14]. 


\subsection{Relaxometric MRI}

Relaxometric MRI can be thought of as the "imaging" version of conventional field-cycling relaxometry. The aim is to obtain spatially-resolved $T_{1}$-dispersion data, by collecting images at a range of evolution magnetic field strengths. The method was first demonstrated by Carlson and colleagues in $1992[15,16]$. These workers placed a small-aperture $(20 \mathrm{~cm}$ gap) pulsed electromagnet in the centre of a $64 \mathrm{mT}$ permanent-magnet clinical MRI system. By energising the electromagnet, they were able to add to or subtract from the permanent magnet's field, thereby changing the field applied to the sample, situated in the centre of the electromagnet. In order to measure a sample's $T_{1}$ at a given evolution magnetic field strength, a spin-warp imaging pulse sequence was repeated 5 times, varying the length of time spent at the evolution field between $50 \mathrm{~ms}$ and $350 \mathrm{~ms}$. In this way it was possible to obtain a set of calculated $T_{1}$ images covering a range of evolution magnetic fields, typically from $20 \mathrm{mT}$ to $110 \mathrm{mT}$. From these data, $T_{1}$ dispersion plots of regions of interest (selected from the images) could be extracted. The disadvantage of the method was the lengthy acquisition time (4 minutes per evolution field value in the dispersion plot). Nevertheless, it was shown that relaxometric images and dispersion plots could be obtained from the extremities and the brains of volunteers [16].

\subsection{Field-Cycled Proton-Electron Double-Resonance Imaging of free radicals}

The method of Proton-Electron Double-Resonance Imaging (PEDRI) of free radicals was first demonstrated in the late 1980s [17]. In PEDRI the Electron Spin Resonance (ESR) of a free radical in solution is irradiated during the acquisition of a proton MR image. In parts of the sample containing the free radical, the Overhauser effect causes a transfer of polarisation from electron to proton spins, resulting in an enhancement of the proton NMR signal, and a consequent increase in image intensity [18]. PEDRI is also known as Overhauser MRI (OMRI), by virtue of its use of the eponymous effect. In a given magnetic field, the ESR frequency is approximately 660 times the proton NMR frequency; therefore, PEDRI is normally implemented at ultra-low field (20 mT) or less, in order to keep the ESR frequency in the bio-compatible range (below $600 \mathrm{MHz}$ ) [19, 20]. Even under these conditions, 
however, there is a potential problem in in vivo small animal studies due to non-resonant absorption of the ESR radiofrequency irradiation. Furthermore, the inherent signal-to-noise ratio (SNR) of the NMR experiment at such low static fields is very low, leading to poor image quality. Field-Cycled PEDRI (FC-PEDRI) was developed in order to counter these problems [18, 21].

Figure 3 shows a typical FC-PEDRI pulse sequence. The magnetic field is switched to a low value during the evolution period (typically $<10 \mathrm{mT}$ ), during which the ESR irradiation is applied at correspondingly low frequency (typically $<300 \mathrm{MHz}$ ). Since the non-resonant absorption of RF power per unit mass (also known as the Specific Absorption Rate, SAR) is proportional to the square of the frequency, a suitable choice of evolution field can reduce the SAR to an acceptable level. Following ESR irradiation, the magnetic field is switched to the higher detection level, for application of the detection NMR RF pulse(s) and imaging gradients. The SNR is mainly determined by the detection magnetic field, so as high a value as possible should be employed. We have built FC-PEDRI systems with detection fields of $59 \mathrm{mT}$ [22] and $450 \mathrm{mT}$ [23].

\section{FFC-MRI magnets}

The main difference between FFC-MRI hardware and that used in conventional MRI is that the former requires the ability to switch rapidly the "static" magnetic field, $B_{0}$, whereas in the latter $B_{0}$ is constant. Naturally, this implies the use of rather special magnet technology in FFC-MRI.

Two main types of magnet system have so far been employed in non-Earth's-field FFC-MRI, namely (i) magnets employing separate, coaxial magnets for polarisation and readout; and (ii) magnets employing constant-field (permanent or superconducting) readout magnets with separate, coaxial, field-offset magnets. These will now be described in turn:

4.1 Magnet systems using separate polarisation and readout magnet coils

In this type of system, the magnet coil providing the polarisation field is energised during the polarisation period (and possibly the evolution period) and is switched off for the remainder of the pulse sequence. Likewise, the readout magnet coil is switched off, except during the readout period. 
An example is the pre-polarised MRI system designed and built by the Stanford University group [7], shown schematically in Figure 4. In their system the readout magnet comprises six discrete coils of conductor, the smallest of which have an inner diameter of $24 \mathrm{~cm}$. The polarisation magnet is coaxial with the readout coils and is formed from a single solenoid with multiple turns of conductor. Its outer and inner diameters are approximately $22 \mathrm{~cm}$ and $11 \mathrm{~cm}$, respectively, making the system suitable for imaging the human wrist. As is typical of this type of system, the readout magnet is designed for high homogeneity (as required for MRI), but relatively low field (24-52 mT). On the other hand, since differences in the polarisation field simply cause "shading" across an image, the polarisation magnet can afford to have quite poor homogeneity, even to the order of $\pm 10 \%$ or more across the field of view. In this system, the polarisation coil can generate a field up to $0.42 \mathrm{~T}$. As stated by Matter et al., a dual concentric design such as this allows the readout magnet to be designed for optimum homogeneity, while the polarising coil can be designed independently in order to optimise bore space and cooling efficiency, at the expense of field homogeneity [7]. Another example of this type of dual magnet system was constructed by the Western Ontario group [24].

\subsection{Dual magnets using field-compensation}

As described by Noack, field-compensation involves "offsetting" the magnetic field of one magnet using a switchable, coaxial magnet coil [1]. Usually the primary magnet operates at constant field, providing the polarization and detection fields, while the variable field of the field-offset magnet enables the evolution field to be selected. In our laboratory we have constructed two FFC-MRI systems using the field-compensation method (both were originally constructed for use with FCPEDRI - see section 3.4 above). The first of these was designed to accommodate samples up to human whole-body size; it employs a $59 \mathrm{mT}$ ferrite permanent magnet to generate the primary magnetic field [22]. The commercial permanent magnet (Field Effects Inc., MA, USA) is of the Halbach design [25], being constructed of a closed "ring" of magnetised ferrite blocks, configured in such a way as to give a homogeneous, vertically-oriented magnetic field within the magnet's bore. The secondary, field-offset coil is a resistive magnet (Magnex Scientific Ltd., UK), wound from copper conductor in a saddle configuration so that its field lies anti-parallel (or parallel) to the 
permanent magnet's field. Figure 5 shows a schematic drawing of the dual magnet, together with a photograph of the system. A significant advantage of this design is that the ferrite primary magnet is non-conductive, so does not support eddy-currents that would otherwise be induced upon switching the field from the field-offset coil. Another advantage is that only the homogeneous, inherently stable magnetic field from the permanent magnet is present during NMR signal readout, since the current in the secondary coil is ramped to zero immediately after the evolution period. The achievable ramp time depends on the maximum voltage delivered by the field-offset magnet's power supply; in this instrument this takes the form of a power-supply amplifier (Copley Controls Inc., MA, USA), and the minimum ramp time (59 $\mathrm{mT}$ to zero or vice versa) is $40 \mathrm{~ms}$ [22].

The second dual-magnet FFC-MRI system constructed in our laboratory makes use of a superconducting magnet (a disused clinical MRI magnet) to provide the polarisation and detection magnetic field of $450 \mathrm{mT}$ [23]. A resistive field-offset coil is again used (Tesla Engineering Ltd., UK), but in this case the coil employs active shielding via a counter-wound coil situated between the offset coil and the cryostat of the superconducting magnet. Active shielding is necessary in order to avoid eddy currents in the cryostat and its radiation shields, and also to avoid inductive coupling with the superconducting magnet coils. Figure 6 shows a schematic drawing of the dual magnet, together with a photograph of the system. Due to the space taken up by the field-offset coil and its active shield, together with the gradient and shim coils, the usable bore diameter in the combined system is reduced to $12 \mathrm{~cm}$; nevertheless, this is sufficient for the study of small imaging phantoms and rodents, for which the system was designed.

\section{Recent developments in FFC-MRI}

\subsection{Protein information via Quadrupole Dips}

Quadrupole dips are reductions in a sample's ${ }^{1} \mathrm{H} T_{1}$ relaxation time which occur at magnetic fields where the Nuclear Quadrupole Resonance (NQR) frequency of coupled quadrupolar nuclei matches the ${ }^{1} \mathrm{H}$ NMR frequency. In a $T_{1}$ dispersion plot they manifest themselves as distinct valleys superimposed on the usual monotonic increase of $T_{1}$ with field strength (or Larmor frequency), hence 
the name "quadrupole dip". (In a plot of $R_{1}=1 / T_{1}$ versus field the effect gives rise to Quadrupole Peaks.) In biological samples the effect is most often observed in immobilised proteins via the quadrupolar ${ }^{14} \mathrm{~N}$ nuclei in amide bonds on the protein backbone. Quadrupole dips were initially studied in the 1970s [26-28] and biological studies began to be carried out in the 1980s [29-32]. Figure 7 shows a dispersion plot from our laboratory, obtained from a sample of cross-linked Bovine Serum Albumin in water; two quadrupole dips can be clearly seen, with centres occurring at the expected fields of $49 \mathrm{mT}$ and $65 \mathrm{mT}$ (the third dip at $16 \mathrm{mT}$ is not seen, due to the range of the data).

The first measurements of quadrupole dips in humans were made by Carlson and colleagues, using a clinical MRI system with a field-offset insert coil (see section 3.3 above) $[15,16]$. They were able to show pronounced quadrupolar $R_{1}$ peaks in the muscles of volunteers, as well as in the pons area of the brain. Lurie also demonstrated quadrupole dips arising from the muscles in a volunteer's arm [33] and from the head [34]. The Aberdeen group also used their whole-body FFC-MRI scanner (section 4.2 above) to generate FFC-MRI images through a volunteer's thighs. A field-cycled inversion-recovery pulse sequence was used to generate images at evolution fields of $57.5 \mathrm{mT}$ (away from a quadrupole dip) and $65 \mathrm{mT}$ (at the centre of a dip). By virtue of the significant difference in $T_{1}$ at these two fields, the images (reproduced in Figure 8) exhibit different intensity in the area of muscle [34, 35].

\subsection{Protein imaging via quadrupole dips}

The work described above showed that immobilised protein could be detected via quadrupole dips, and demonstrated the potential for protein-dependent contrast in FFC-MR images, via the effect. The natural next step is to develop the method into a technique capable of quantifying protein. The rationale for protein quantitation via quadrupolar cross-relaxation was first shown by Jiao and Bryant, who demonstrated that the "height" of a quadrupole peak in $R_{1}$ is directly proportional to the concentration of immobilised protein in the sample [36]. The height of the peak, $\Delta R_{1}$, is taken as the difference between the measured $R_{1}$ value and the value of the relaxation rate, $R_{1}{ }^{0}$ that would have been measured in the absence of the ${ }^{14} \mathrm{~N}-{ }^{1} \mathrm{H}$ cross-relaxation. $R_{1}{ }^{0}$ can be estimated by fitting the dispersion curve's "background" (i.e. beyond and between the quadrupole peaks) to an appropriate 
model then interpolating the background relaxation rate value at the peak position. Jiao and Bryant modelled the background relaxation as $R_{1}{ }^{0}=\mathrm{A} v^{-0.5}$ where $\mathrm{A}$ is a constant and $v$ is the proton Larmor frequency [36]).

A similar approach can be adopted in order to produce quantitative $\left(\Delta R_{1}\right)$ protein-concentration images. In our laboratory we demonstrated such a method, using a heat-treated hen's egg as a test sample [37]. Upon heating, the albumin protein in the egg "white" denatures and becomes crosslinked, and the immobilised protein gives rise to pronounced quadrupole peaks (see Figure 9a). In order to generate a $\Delta R_{1}$ image, three calculated- $R_{1}$ images were obtained, using a field-cycling inversion-recovery pulse sequence, at evolution fields of $56 \mathrm{mT}, 65 \mathrm{mT}$ and $75 \mathrm{mT}$. The lowest and highest fields correspond to the edges of the quadrupole peak centred on $65 \mathrm{mT}$, and allow $R_{1}{ }^{0}$ at 65 $\mathrm{mT}$ to be estimated by interpolation. Following data collection and calculation of the $R_{1}$ and $R_{1}{ }^{0}$ maps, a calculated $\Delta R_{1}$ map was obtained, as shown in Figure 9b. The egg "white" region shows a relatively uniform intensity, with average $\Delta R_{1}=0.68 \mathrm{~s}^{-1}$, indicating homogeneous distribution of immobilised protein. The egg yolk region comprises primarily noise, with average $\Delta R_{1}=-0.02 \mathrm{~s}^{-1}$, consistent with the fact that the yolk does not exhibit quadrupole peaks. $T_{1}$-dispersion field-cycling MRI, exploiting quadrupolar cross-relaxation, has also been reported by Ungersma et al., who showed that protein contrast could be obtained in test objects and in the extremities of human volunteers [38-40].

\subsection{Protein quantification in a model biological system}

Quadrupole dips (peaks) offer a non-invasive method of quantifying immobilised protein, and could find use in many areas of biology and medicine. For example, diseases such as Parkinson's Disease and Alzheimer's Disease involve malformed proteins within plaques in the brains of sufferers; ultimately, it may be possible to diagnose and characterise these diseases via their quadrupole-dip signatures. Many physiological processes involve protein reactions, and one of these which is well understood is thrombosis - the formation of blood clots. Since thrombosis involves the cross-linking of fibrinogen protein to form fibrin, it is reasonable to assume that the process might be detectable through the measurement of quadrupole dips, and that the measurements ought to be able to quantify 
the clotting process in some way. Furthermore, the basic process of formation of a fibrin clot is readily modelled in the laboratory, making it an ideal, controllable system to study by field-cycling relaxometry.

In our laboratory we have conducted pilot studies to investigate by NMR relaxometry the formation of fibrin by the thrombin enzyme mediated cleavage of fibrinogen $[41,42]$. This involved preparing samples of human fibrinogen (Hyphen BioMed, France) at concentrations varying from 0.1 to $10 \mathrm{mg} / \mathrm{mL}$. Agarose was added to the samples in order ensure homogeneity of the clots. Clotting was initiated, in standard NMR sample tubes, by adding thrombin (Sigma-Aldrich, UK) at a concentration of $0.4 \mathrm{U} / \mathrm{mL}$. Following the formation of model clots, $R_{1}$ dispersion plots were measured using a commercial relaxometer (“SMARtracer”, Stelar S.r.1., Italy). Dispersion plots were fitted using a program written in MATLAB (Mathworks, Cambridge, MA, USA) which employed Lorentzian bells for the quadrupole peaks, superimposed on a power-law background curve. This allowed the height of the quadrupole peaks ( $\Delta R_{1}$, as described in section 5.2), to be estimated. Figure 10a shows a typical dispersion plot and its fitted curve, while Figure 10b shows a plot of $\Delta R_{1}$ versus fibrinogen concentration. It can be seen that there is a linear relationship between the height of the quadrupole peak and the concentration of fibrinogen (in fact, the concentration of fibrin in the clot). The detection threshold estimated from these data is $1.04 \mathrm{mg} / \mathrm{mL}$, significantly less than the typical concentration of fibrinogen in human plasma of $2-4 \mathrm{mg} / \mathrm{mL}$. This early work indicates that FFC relaxometry could provide useful information in the study of thrombosis, while FFC-MRI might provide additional diagnostic information in the clinic.

\subsection{FFC-MRI with contrast agents}

Another means of exploiting the benefits of field-cycling in MRI is through the use of relaxationenhancing contrast agents. The idea here is to use field-cycling to make use of the fact that many contrast agents exhibit radically different relaxivity at different magnetic fields. Therefore, by collecting one FFC-MR image at an evolution field where the agent has low relaxivity, and another image at an evolution field corresponding to high relaxivity, it should be possible to increase the 
sensitivity of detection of a contrast agent enhanced FFC-MR experiment. A variant of this method, dubbed Delta Relaxation Enhanced MR (DREMR), uses field-cycling to obtain image contrast based on the slope of a contrast agent's dispersion curve, in the clinically-used magnetic field range (1.5 T) [43].

We are investigating the use of contrast agents tailored for use with FFC-MRI at low magnetic field. One such agent consists of liposomes encapsulating $\mathrm{Mn}(\mathrm{II})$ ions $\left(\mathrm{as} \mathrm{MnCl}_{2}\right)$ in their internal aqueous cavity [44]. This system exhibits significant differences in relaxivity between $5 \mathrm{mT}$ and $60 \mathrm{mT}$, as shown in Figure 11a; by comparison, an aqueous sample of $\mathrm{CuSO}_{4}$ exhibits almost no difference in $R_{1}$ over the same field range. Pilot experiments have been conducted in order to investigate the potential of Mn(II) liposomes as FFC-MRI contrast agents, with experiments conducted on our $59 \mathrm{mT}$ wholebody FFC-MRI system [22]. A field-cycling inversion-recovery pulse sequence was used to generate calculated $R_{1}$ images at evolution fields of $5 \mathrm{mT}$ and $59 \mathrm{mT}$. From these data a $\Delta R_{1}$ image was produced (Figure 11b). The liposomes used here (111 nm average diameter) contained approximately $10^{3} \mathrm{Mn}(\mathrm{II})$ ions each. A suspension containing $0.06 \mathrm{mM} \mathrm{Mn}(\mathrm{II})$ ions corresponds to $30 \mathrm{nM}$ concentration of liposomes (determined by fluorimetric measurement, which was in agreement with a calculation by the method of Epstein et al. [45]). This sample was readily detectable in the $\Delta R_{1}$ image, indicating that the use of FFC-MRI with the tailored liposomal agent does indeed give rise to high sensitivity of detection. More recently, we have found that the attainable sensitivity can be further markedly improved by suitable formulation of the liposome membrane components and the use of more concentrated Mn(II)-hydration solutions. The detection sensitivities of these systems are well in the range of those required for Molecular Imaging applications. The possibility of targeting liposomes that act as high-sensitivity paramagnetic particles will allow the design of application protocols involving the use of $\mathrm{Mn}$ (II) ions at concentrations well below those that may give rise to toxicity concerns. Thus such a class of FFC-MRI contrast agents may represent a new class of very powerful and innovative molecular imaging probe.

Whereas our work is concentrated on the use of FFC-MRI at low field, in their DREMR work the group at the University of Western Ontario have used field cycling over a range of fields centred on 
1.5 T; thus, their technique is potentially applicable on current clinical MR systems, albeit with the addition of extra "field-offset" coil hardware to switch the magnetic field [43]. Alford et al. inserted a field-offset period at the start of a spin-echo imaging sequence. By collecting images with the offset set at $-70 \mathrm{mT}$ then $+70 \mathrm{mT}$ they were able to exploit differences in samples' $R_{1}$ values over the range $1.43-1.57 \mathrm{~T}$. In their recent publication these authors used the commercial albumin-binding contrast agent "Vasovist", and conducted in vitro experiments with different concentrations of the agent bound to rabbit serum albumin (RSA) in solution. Since the relaxivity of the RSA-bound Vasovist is highly field-dependent, Alford et al. were able to show that the DREMR method was able to detect the bound agent at concentrations as low as $10 \mu \mathrm{M}$ of Vasovist [43].

The above work demonstrates that FFC-MRI has significant potential to increase the sensitivity and specificity of contrast-enhanced MR studies.

\subsection{Localised image-guided relaxometry}

As discussed above, there are potential advantages in collecting relaxation dispersion curves of tissues, and the natural progression is to collect dispersion data of humans, in vivo. While it is relatively straightforward to collect a dispersion curve from the whole volume of tissue inside an RF receiver coil [33], the disadvantage of that approach is that the relaxation time data inevitably represents an average of the multitude of tissue types within the coil's sensitive volume. What is needed is a method of selecting a region of interest from an image of the body, then obtaining a dispersion plot from that well-defined location. One approach is that adopted by Carlson et al., who obtained a large number of calculated $R_{1}$ images at a range of evolution field strengths, from which dispersion plots of selected regions could be obtained $[15,16]$. While that method was effective, its major disadvantage was the time necessary to collect the data (typically 2 hours for a 32 field-point dispersion curve), which would be unacceptable in a clinical setting.

We have implemented an alternative method of localised dispersion curve measurement, which we call Localised Image-Guided Relaxometry. In this method pilot images are first collected, then a region-of-interest is drawn on the images. The FFC-MRI scanner's software then runs a field-cycling 
relaxometry pulse sequence in order to collect a dispersion curve from only the selected region of the sample [46, 47]. Spatial selection of the region for relaxometry is achieved by the use of a PRESS-like method, where a selective $90^{\circ}$ pulse followed by two successive selective $180^{\circ}$ pulses, each pulse being applied in the presence of orthogonal magnetic field gradients [48]. The localised image-guided relaxometry pulse sequence is shown in Figure 12, while Figure 13 shows a pilot image of a volunteer's thighs together with a $T_{1}$ dispersion plot obtained from a region of muscle; quadrupole dips can clearly be seen, centred on the expected fields of $49 \mathrm{mT}$ and $65 \mathrm{mT}$. In this case, the pilot image took 2.5 min to collect, while a typical collection time for a 40 field-point dispersion curve $(\mathrm{NEX}=2)$ is $5 \mathrm{~min}$.

\subsection{Magnetisation Transfer Contrast FFC-MRI}

Magnetisation Transfer Contrast (MTC) is a standard technique in the armoury of MRI, and is widely used in clinical MRI in order to elucidate information pertaining to macromolecules in the body (which are not usually detected directly due to their very broad NMR lines, or equivalently their very short $T_{2}$ relaxation times) [49]. MTC MRI is typically implemented by incorporating a period of offresonance RF irradiation into the pulse sequence, in order to partially saturate the broad-line (10s of $\mathrm{kHz})$ macromolecule-bound resonance while not affecting directly the narrow-line (10s of $\mathrm{Hz})$ waterproton resonance. After irradiation, magnetisation is transferred between the macromolecular-bound proton "pool" and the free water "pool", leading to a reduction in intensity of the detected NMR signals. While there have been many studies of MTC at medium and high magnetic field ( $0.5 \mathrm{~T}$ and above), there is a dearth of information in the literature concerning MTC at ultra-low or low magnetic field, and very little concerning the variation of the MT effect with magnetic field (especially at low field). We have recently shown that it is possible to combine MTC with FFC-MRI in order to provide a new method of producing off-resonance irradiation of bound-pool spins [50, 51]; the method should also be applicable to explore the MT effect as a function of magnetic field strength.

One difficulty of implementing MTC at low or ultra-low field is associated with the need for offresonance irradiation. This necessitates applying RF (continuous-wave or pulsed) at a frequency that 
may be several 10s of $\mathrm{kHz}$ distant from the fundamental water proton Larmor frequency (to which the RF coil must be tuned). The problem arises because the coil's response will fall off as the frequency departs from resonance, making it difficult to deliver a precisely controlled RF field for MT irradiation. For example, an RF coil operating at $3 \mathrm{MHz}$ which has a Q-factor of 300 will have an inherent bandwidth of $10 \mathrm{kHz}$, making irradiation outside that band (as needed for MTC) problematic. Magnetic field cycling can completely alleviate this problem, by applying the MT irradiation at the same irradiation frequency, but at a main magnetic field offset by an appropriate amount. For example, if a $20 \mathrm{kHz}$ offset frequency is desired, the field offset required would be $20 \mathrm{kHz} * 2 \pi / \gamma=$ $0.468 \mathrm{mT}$ (where $\gamma$ is the proton gyromagnetic ratio). Our work has shown that MT "Z-spectra" (ratio of signal with and without MT irradiation, as a function of offset frequency) obtained using the FFC field-offset MT method are identical to spectra obtained by the conventional frequency-offset technique $[50,51]$. We have also shown that images with MT contrast can be obtained using the new method, by incorporating field-offset with MT irradiation into an imaging pulse sequence.

\section{Conclusions}

This article has explored some key aspects of the emerging field of fast field-cycling magnetic resonance imaging, which has applications as diverse as imaging free radicals, monitoring immobilised protein, combining PET and MRI, as well as imaging in the Earth's magnetic field. In the biomedical realm, the ability to detect immobilised protein has many potential applications, especially in light of the huge variety of conditions and diseases that involve protein malformation or mis-folding, including Parkinson's Disease, Alzheimer's Disease, and Variant Creutzfeldt-Jacob Disease (CJD), to name but three. The use of contrast agents (for example, Mn(II)-liposomes) specially tailored for use with FFC-MRI offers the potential for significant improvements in sensitivity and specificity, leading to molecular imaging applications.

We are currently working on the use of FFC relaxometry and FFC-MRI in a number of disease models, and hope to expand the scope of this work over the coming years. In addition we, and others, are building new FFC-MRI systems that promise greater flexibility and sensitivity. With more 
research groups gradually joining the field, it can be expected that the techniques and applications of FFC-MRI will continue to expand.

\section{Acknowledgements}

The authors acknowledge financial support for the FFC-MRI project from Research Councils UK and the Engineering and Physical Sciences Research Council, under the Basic Technology scheme (grant no. EP/E036775/1). 


\section{References}

[1] F. Noack, NMR field-cycling spectroscopy - principles and applications, Prog.Nucl.Magn.Reson.Spectrosc., 18 (1986) 171-276.

[2] E. Anoardo, G. Galli and G. Ferrante, Fast-field-cycling NMR: Applications and instrumentation, Appl. Magn. Reson., 20 (2001) 365-404.

[3] R. Kimmich and E. Anoardo, Field-cycling NMR relaxometry, Prog.Nucl.Magn.Reson.Spectrosc., 44 (2004) 257-320.

[4] G. Ferrante and S. Sykora, Technical aspects of fast field cycling, Adv.Inorg.Chem., 57 (2006) 405-470.

[5] M. Packard and R. Varian, Free nuclear induction in the earth's magnetic field, Phys.Rev., A93 (1954) 941.

[6] A. Macovski and S. Conolly, Novel approaches to low-cost MRI, Magn.Reson.Med., 30 (1993) 221-230.

[7] N.I. Matter, G.C. Scott, T. Grafendorfer, A. MacOvski and S.M. Conolly, Rapid polarizing field cycling in magnetic resonance imaging, IEEE Trans.Med.Imaging, 25 (2006) 84-93.

[8] R.D. Venook, N.I. Matter, M. Ramachandran, S.E. Ungersma, G.E. Gold, N.J. Giori, A. Macovski, G.C. Scott and S.M. Conolly, Prepolarized magnetic resonance imaging around metal orthopedic implants, Magn.Reson.Med., 56 (2006) 177-186.

[9] K.M. Gilbert, W.B. Handler, T.J. Scholl, J.W. Odegaard and B.A. Chronik, Design of field-cycled magnetic resonance systems for small animal imaging, Phys.Med.Biol., 51 (2006) 2825-2841.

[10] H. Peng, W.B. Handler, T.J. Scholl, P.J. Simpson and B.A. Chronik, Proof-of-principle study of a small animal PET/field-cycled MRI combined system using conventional PMT technology, Nucl.Instrum.Methods Phys.Res.Sect. A, 612 (2010) 412-420.

[11] J. Stepisnik, V. Erzen and M. Kos, NMR imaging in the earth's magnetic field, Magn. Reson. Med., 15 (1990) 386-391.

[12] A. Mohoric, G. Planinsic, M. Kos, A. Duh and J. Stepisnik, Magnetic resonance imaging system based on earth's magnetic field, Instrum Sci Technol, 32 (2004) 655-667.

[13] M.E. Halse, A. Coy, R. Dykstra, C. Eccles, M. Hunter, R. Ward and P.T. Callaghan, A practical and flexible implementation of 3D MRI in the earth's magnetic field, J.Magn.Reson., 182 (2006) 7583.

[14] N. Kelso, S.-K. Lee, L.-S. Bouchard, V. Demas, M. Mück, A. Pines and J. Clarke, Distortion-free magnetic resonance imaging in the zero-field limit, J.Magn.Reson., 200 (2009) 285-290.

[15] J.W. Carlson, L.E. Crooks, M. Arakawa, D.M. Goldhaber, D.M. Kramer and L. Kaufman, Switched-field magnetic resonance imaging, SPIE Medical Imaging VI: Instrumentation, 1651 (1992) 22-27.

[16] J.W. Carlson, D.M. Goldhaber, A. Brito and L. Kaufman, MR relaxometry imaging - work in progress, Radiology, 184 (1992) 635-639. 
[17] D.J. Lurie, D.M. Bussell, L.H. Bell and J.R. Mallard, Proton-electron double magnetic resonance imaging of free radical solutions, J.Magn.Reson., 76 (1988) 366-370.

[18] D.J. Lurie, "Proton-Electron Double-Resonance Imaging (PEDRI)," in In Vivo EPR (ESR): Theory and Applications, vol. 18, L.J. Berliner Ed. New York: Kluwer Academic / Plenum Publishers, pp. 547-578 (2003).

[19] D.J. Lurie, I. Nicholson, M.A. Foster and J.R. Mallard, Free-radicals imaged in vivo in the rat by using proton electron double-resonance imaging, Philos.Trans.R.Soc.Lond.Ser.A-Math.Phys.Eng.Sci., 333 (1990) 453-456.

[20] D.J. Lurie, H.H. Li, S. Petryakov and J.L. Zweier, Development of a PEDRI free-radical imager using a 0.38 T clinical MRI system, Magn.Reson.Med., 47 (2002) 181-186.

[21] D.J. Lurie, J.M.S. Hutchison, L.H. Bell, I. Nicholson, D.M. Bussell and J.R. Mallard, Fieldcycled proton electron double-resonance imaging of free radicals in large aqueous samples, J.Magn.Reson., 84 (1989) 431-437.

[22] D.J. Lurie, M.A. Foster, D. Yeung and J.M.S. Hutchison, Design, construction and use of a largesample field-cycled PEDRI imager, Phys.Med.Biol., 43 (1998) 1877-1886.

[23] D.J. Lurie, G.R. Davies, M.A. Foster and J.M.S. Hutchison, Field-cycled PEDRI imaging of free radicals with detection at $450 \mathrm{mT}$, Magn.Reson.Imaging, 23 (2005) 175-181.

[24] K.M. Gilbert, T.J. Scholl, W.B. Handler, J.K. Alford and B.A. Chronik, Evaluation of a positron emission tomography (PET)-compatible field-cycled MRI (FCMRI) scanner, Magn.Reson.Med., 62 (2009) 1017-1025.

[25] K. Halbach, Design of permanent multipole magnets with oriented rare earth cobalt material, Nucl.Instrum.Methods, 169 (1980) 1-10.

[26] J. Seliger, R. Osredkar, M. Mali and R. Blinc, ${ }^{14} \mathrm{~N}$ quadrupole resonance of some liquid crystalline compounds in the solid, J.Chem.Phys., 65 (1976) 2887-2891.

[27] G. Voigt and R. Kimmich, Quadrupolar dip in proton relaxation dispersion of poly(vinyl chloride), J.Magn.Reson., 24 (1976) 149-154.

[28] R. Kimmich, Nuclear magnetic relaxation in the presence of quadrupole nuclei, Z.Naturforsch., 32a (1977) 544-554.

[29] R. Kimmich, Field cycling in NMR relaxation spectroscopy: Applications in biological, chemical and polymer physics Bulletin of Magnetic Resonance, 1 (1980) 195.

[30] R. Kimmich, W. Nusser and F. Winter, In vivo NMR field-cycling relaxation spectroscopy reveals 14N1H relaxation sinks in the backbones of proteins. Phys.Med.Biol., 29 (1984) 593-596.

[31] R. Kimmich, F. Winter, W. Nusser and K.H. Spohn, Interactions and fluctuations deduced from proton field-cycling relaxation spectroscopy of polypeptides, dna, muscles, and algae, J.Magn.Reson., 68 (1986) 263-282.

[32] P.A. Rinck, H.W. Fischer, L. Vander Elst, Y. Van Haverbeke and R.N. Muller, Field-cycling relaxometry: Medical applications, Radiology, 168 (1988) 843-849. 
[33] D.J. Lurie, "Quadrupole-Dip Measurement in Humans by Whole-Body Field-Cycling NMR Relaxometry and Imaging", in Proc. 29th Ampere and 13th ISMAR Conference, Berlin, Germany, pp. 254 (1998).

[34] D.J. Lurie, "Field-Cycled Magnetic Resonance Imaging - Techniques and Applications", in Symposium on Field-Cycling NMR Relaxometry, Berlin, Germany, pp. 5 (1998).

[35] D.J. Lurie, "Quadrupole Dips Measured by Whole-Body Field-Cycling Relaxometry and Imaging", in Proc. Int. Society of Magnetic Resonance in Medicine 7th Meeting, Philadelphia, USA, pp. 653 (1999).

[36] X.Q. Jiao and R.G. Bryant, Noninvasive measurement of protein concentration, Magn.Reson.Med., 35 (1996) 159-161.

[37] G.R. Davies and D.J. Lurie, "Quantitative Field-Cycling $T_{1}$ Dispersion Imaging", in Proceedings of the ISMRM 13th Scientific Meeting, Miami Beach, USA, pp. 2187 (2005).

[38] S. Ungersma, N. Matter, A. Macovski, S. Conolly and G. Scott, "In Vivo MR Imaging with $T_{1}$ Dispersion Contrast", in Proceedings of the ISMRM 13th Scientific Meeting, Miami Beach, USA, pp. 414 (2005).

[39] S.E. Ungersma, N.I. Matter, J.W. Hardy, R.D. Venook, A. Macovski, S.M. Conolly and G.C. C. Scott, Magnetic resonance imaging with $\mathrm{T}_{1}$ dispersion contrast , Magn.Reson.Med., 55 (2006) 1362-1371.

[40] S. Ungersma, N. Matter, A. Macovski, S. Conolly and G. Scott, "MR Imaging with $T_{1}$ Dispersion Contrast", in Proceedings of the ISMRM 12th Scientific Meeting, Kyoto, Japan, pp. 179 (2004).

[41] S. Ismail, L.M. Broche, N.A. Booth and D.J. Lurie, "Detection of fibrin by Fast Field-Cycling magnetic resonance techniques", in $6^{\text {th }}$ Conference on Field-Cycling NMR Relaxometry, Turin, Italy, pp. 14 (2009).

[42] S. Ismail, L.M. Broche, N.A. Booth and D.J. Lurie, "Non-invasive Measurement of Fibrin Concentration by Fast Field-Cycling NMR Technique", in Proceedings of the ISMRM 18th Scientific Meeting, Stockholm, Sweden, pp. 915 (2010).

[43] J.K. Alford, B.K. Rutt, T.J. Scholl, W.B. Handler and B.A. Chronik, Delta relaxation enhanced mr: Improving activation - specificity of molecular probes through R1 dispersion imaging, Magnetic Resonance in Medicine, 61 (2009) 796-802.

[44] D. O Hogain, G.R. Davies, S. Baroni, G. Ferrante, S. Aime and D.J. Lurie, "Use of Contrast Agents with Fast Field-Cycling MRI”, in Italian Chapter of ISMRM, Milan, Italy, pp. 56 (2010).

[45] H. Epstein, E. Afergan, T. Moise, Y. Richter, Y. Rudich and G. Golomb, Number-concentration of nanoparticles in liposomal and polymeric multiparticulate preparations: Empirical and calculation methods, Biomaterials, 27 (2006) 651-659.

[46] K.J. Pine, G.R. Davies and D.J. Lurie, "Localized In Vivo Field-Cycling Relaxometry", in Proceedings of the ISMRM 17th Scientific Meeting, Honolulu, USA, pp. 2743 (2009).

[47] K.J. Pine, G.R. Davies and D.J. Lurie, Field-cycling NMR relaxometry with spatial selection, Magn.Reson.Med., In Press (2010) 
[48] P.A. Bottomley, Spatial localization in NMR spectroscopy in vivo, Ann.New York Acad.Sci., 508 (1987) 333-348.

[49] S.D. Wolff and R.S. Balaban, Magnetization transfer contrast (MTC) and tissue water proton relaxation in vivo, Magn.Reson.Med., 10 (1989) 135-144.

[50] C.-H. Choi, G.R. Davies and D.J. Lurie, "Off-Resonance Magnetisation Transfer Contrast MRI using Fast Field-Cycling Technique", in Proceedings of the ISMRM 17th Scientific Meeting, Honolulu, USA, pp. 2747 (2009).

[51] C.-H. Choi, G.R. Davies and D.J. Lurie, Off-resonance magnetisation transfer contrast (MTC) MRI using fast field-cycling (FFC), J.Magn.Reson., 204 (2010) 145-149. 


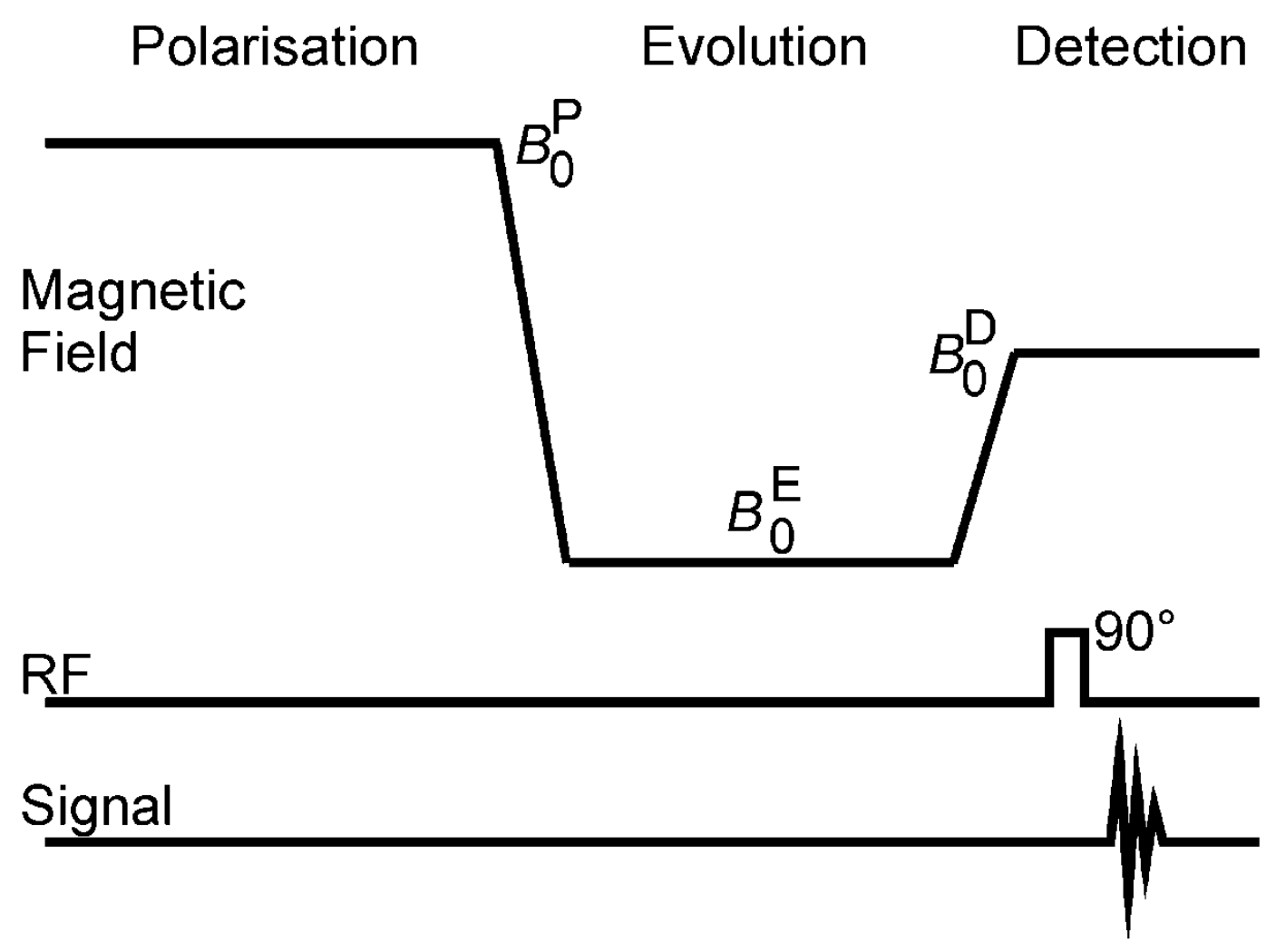

Figure 1: Typical Fast Field-Cycling NMR pulse sequence. 


\section{Pre-Polarization Readout (Detection)}

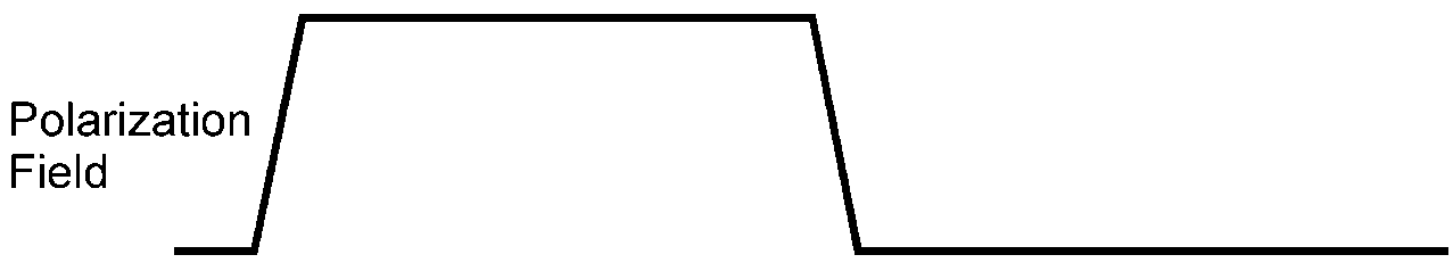

Readout

Field

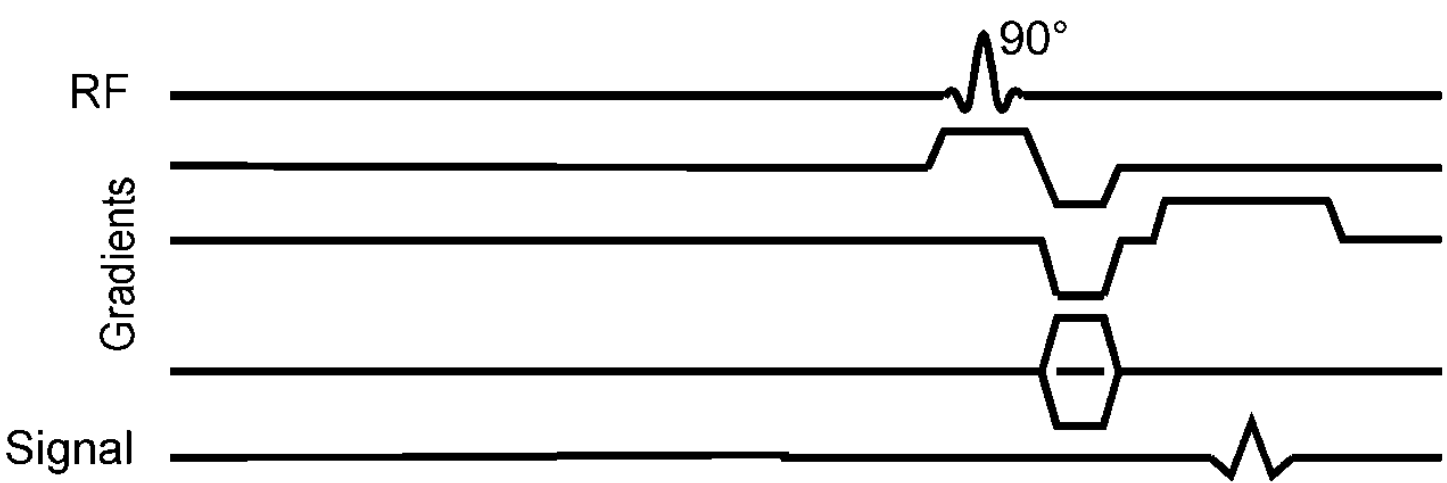

Figure 2: Example of pre-polarized MRI pulse sequence. The Polarization and Readout magnetic fields are generated by separate, coaxial, magnet coils. 


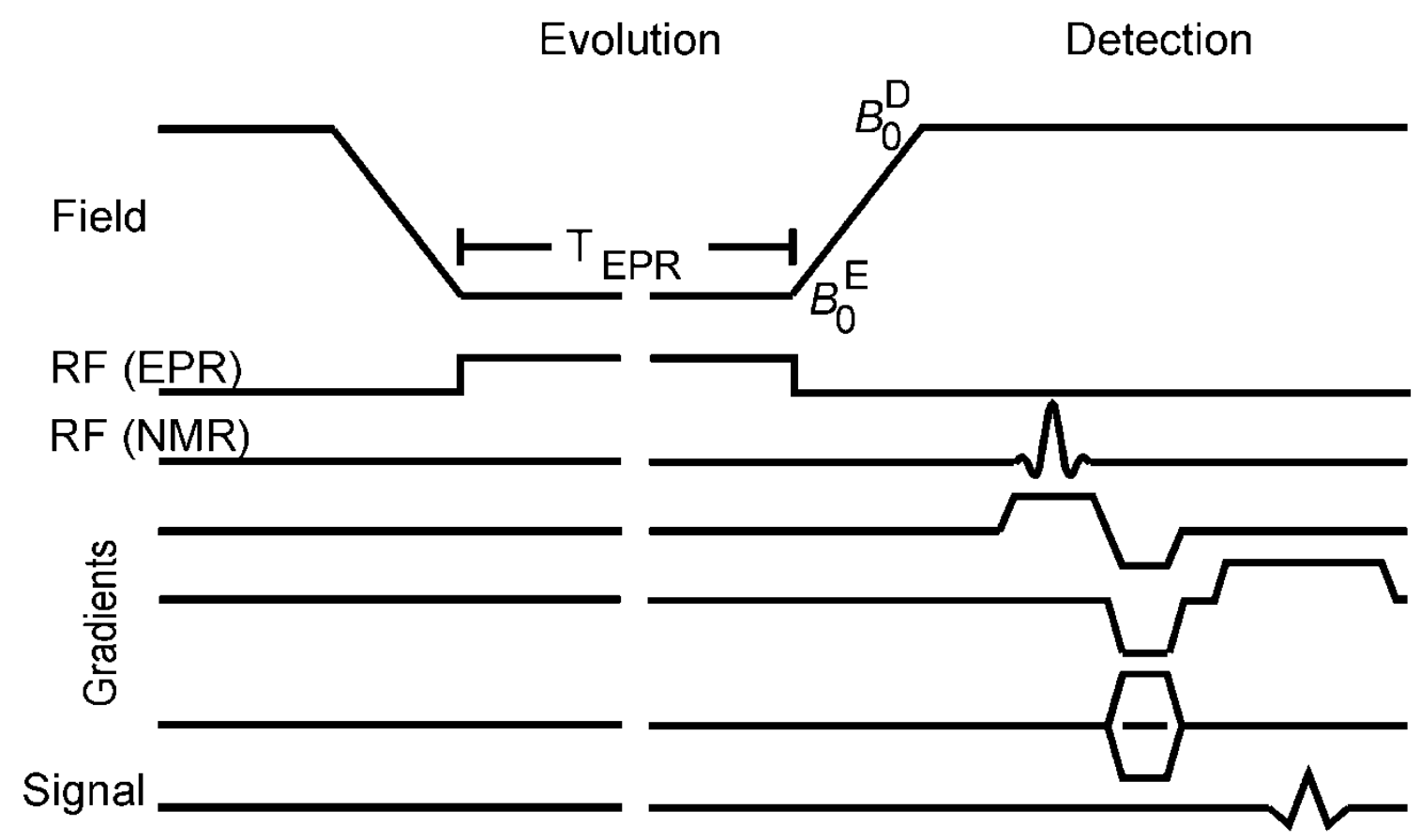

Figure 3: Field-Cycled PEDRI (FC-PEDRI) pulse sequence, for imaging free radicals. 


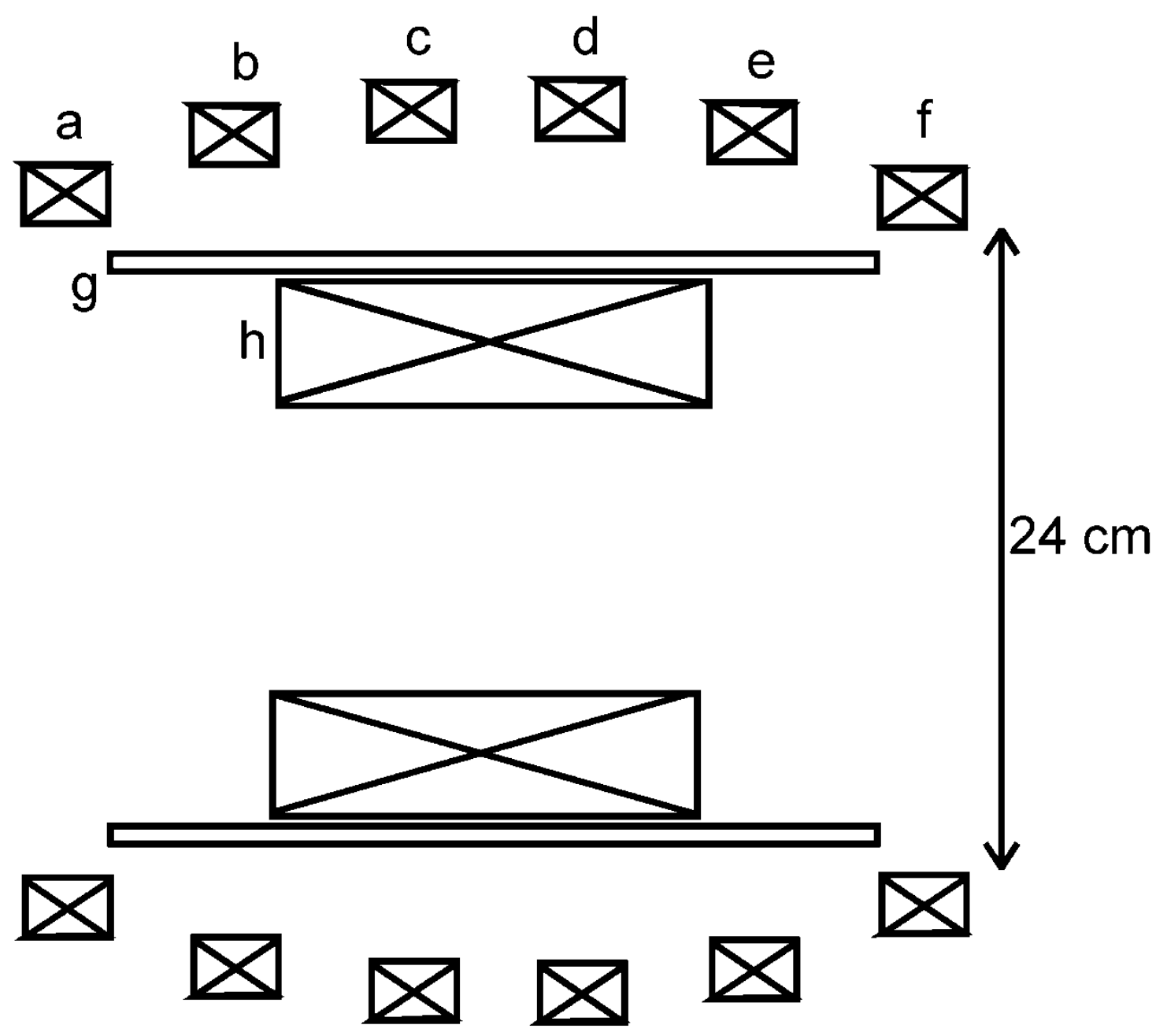

Figure 4: Diagram showing cross-section through pre-polarized MRI magnet constructed by the Stanford group. a-f are coils of the readout magnet; $g$ represents the gradient coils; $h$ is the prepolarizing magnet coil. 

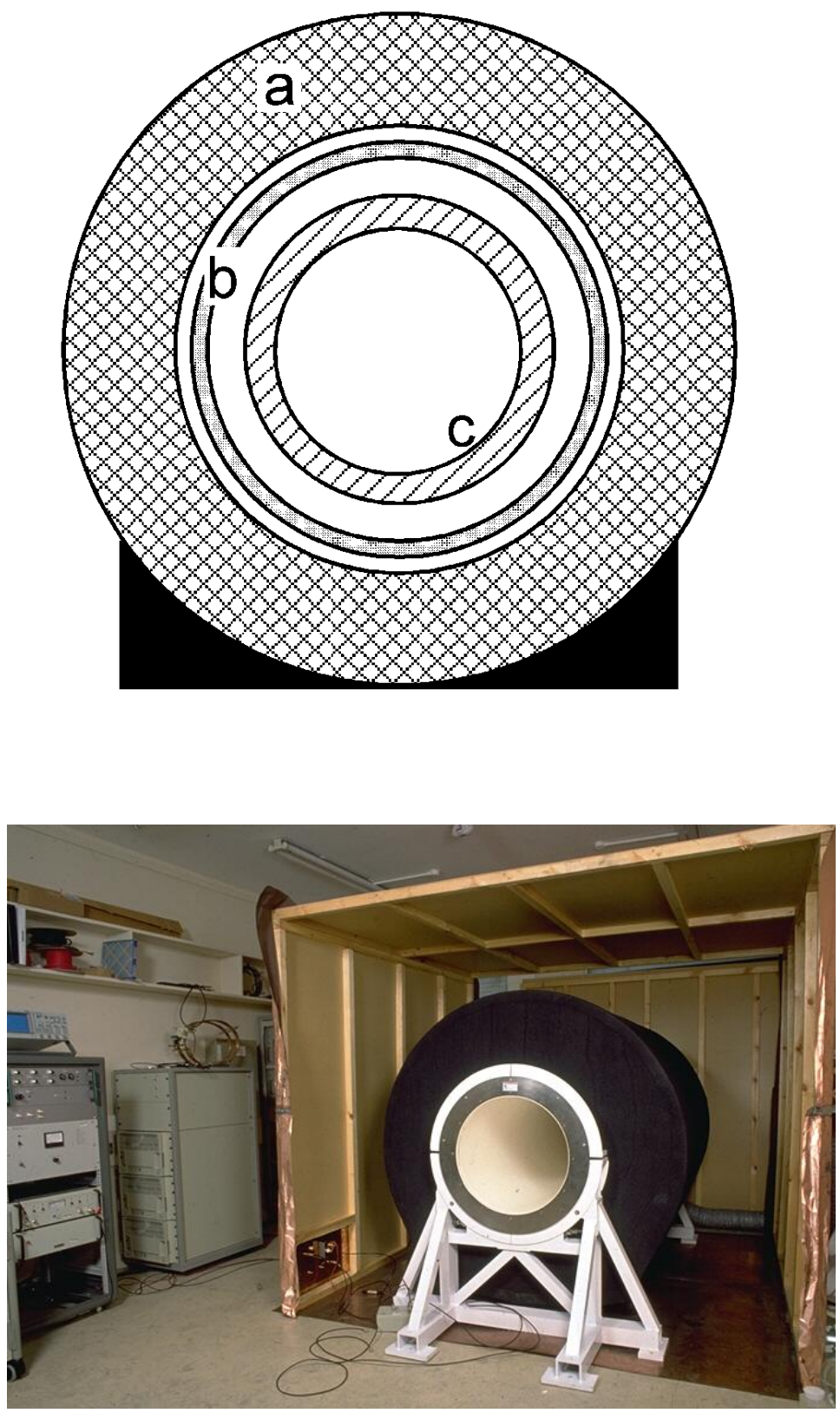

Figure 5: Left - schematic diagram showing end-view of whole-body FFC-MRI magnet system. a:$59 \mathrm{mT}$ permanent magnet generating vertical field; b:- gradient coils (X, Y and Z); c:- saddle configuration resistive field-offset coil, generating vertical field up to $59 \mathrm{mT}$ (anti-parallel or parallel to field of permanent magnet). Right - Photograph of whole-body FFC-MRI magnet system, prior to completion of the copper Faraday cage (part of which can be seen, on its wooden frame). 

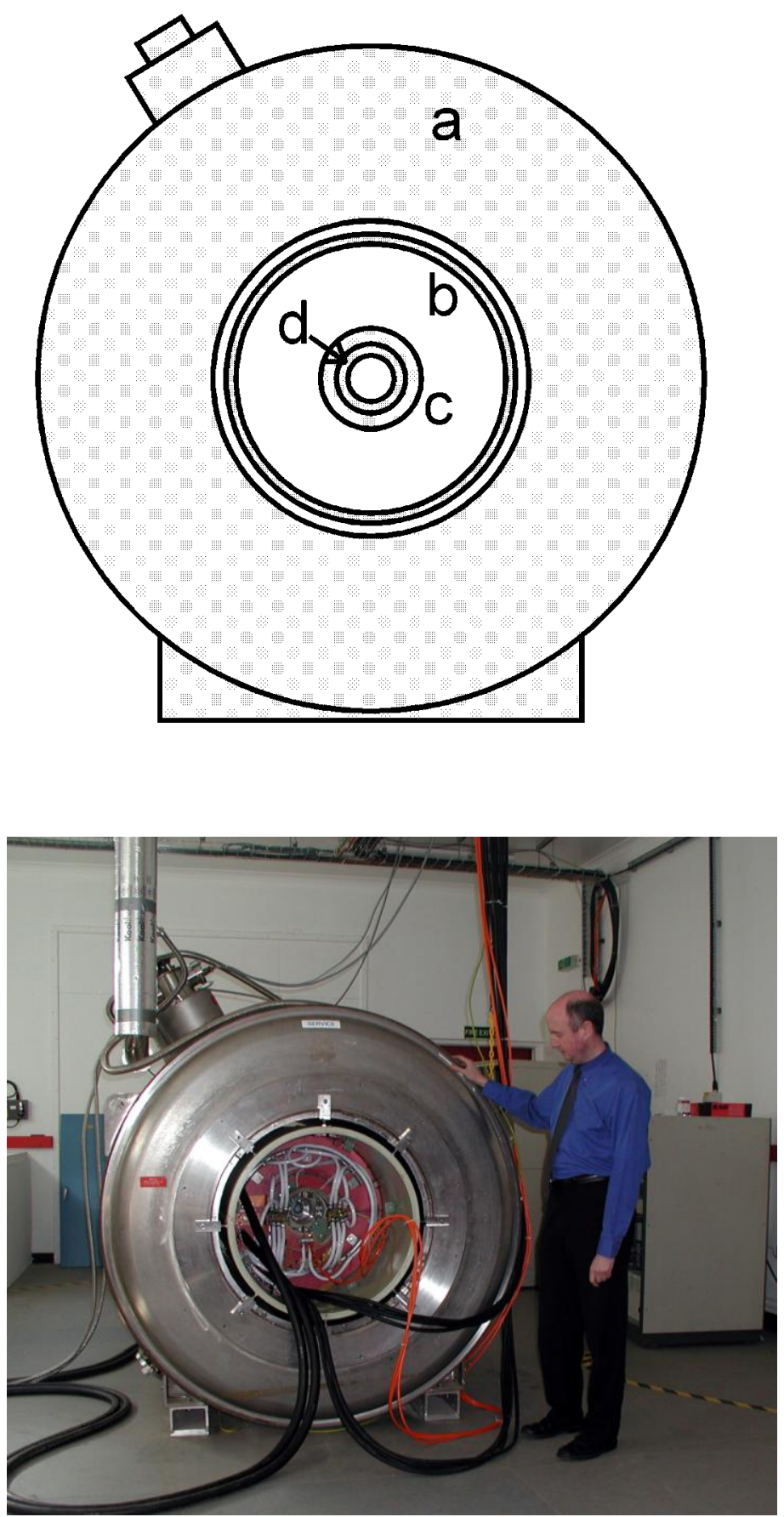

Figure 6: Left - schematic diagram showing end-view of $450 \mathrm{mT}$ FFC-MRI magnet system. a:- 450 $\mathrm{mT}$ superconducting magnet generating field out of page; b:- active shield coil; c:- solenoidal configuration resistive field-offset coil, generating field up to $450 \mathrm{mT}$ (anti-parallel or parallel to field of superconducting magnet); d:- gradient and shim coils. Right - photograph of $450 \mathrm{mT}$ FFC-MRI magnet system, next to one of the authors (DJL). 


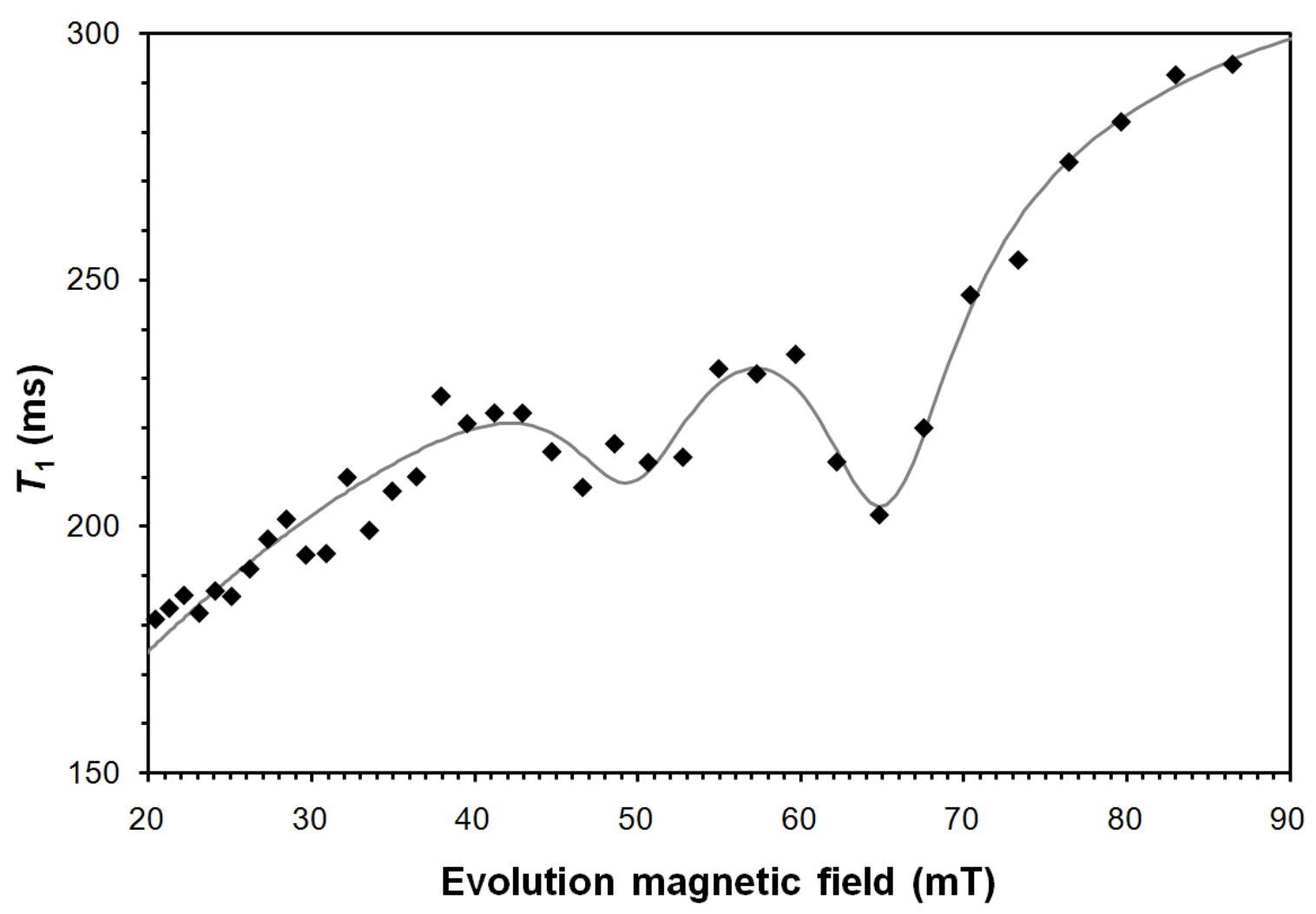

Figure 7: $T_{1}$ dispersion plot of sample of BSA gel (16.5\% w/v in water), showing quadrupole dips centred on $49 \mathrm{mT}$ and $65 \mathrm{mT}$. Measurement conducted on benchtop relaxometer ("SMARtracer", Stelar S.r.l., Italy). Diamonds - experimental data. Solid line - fit to $R_{1}$ using power-law background and Lorentzian peaks. 

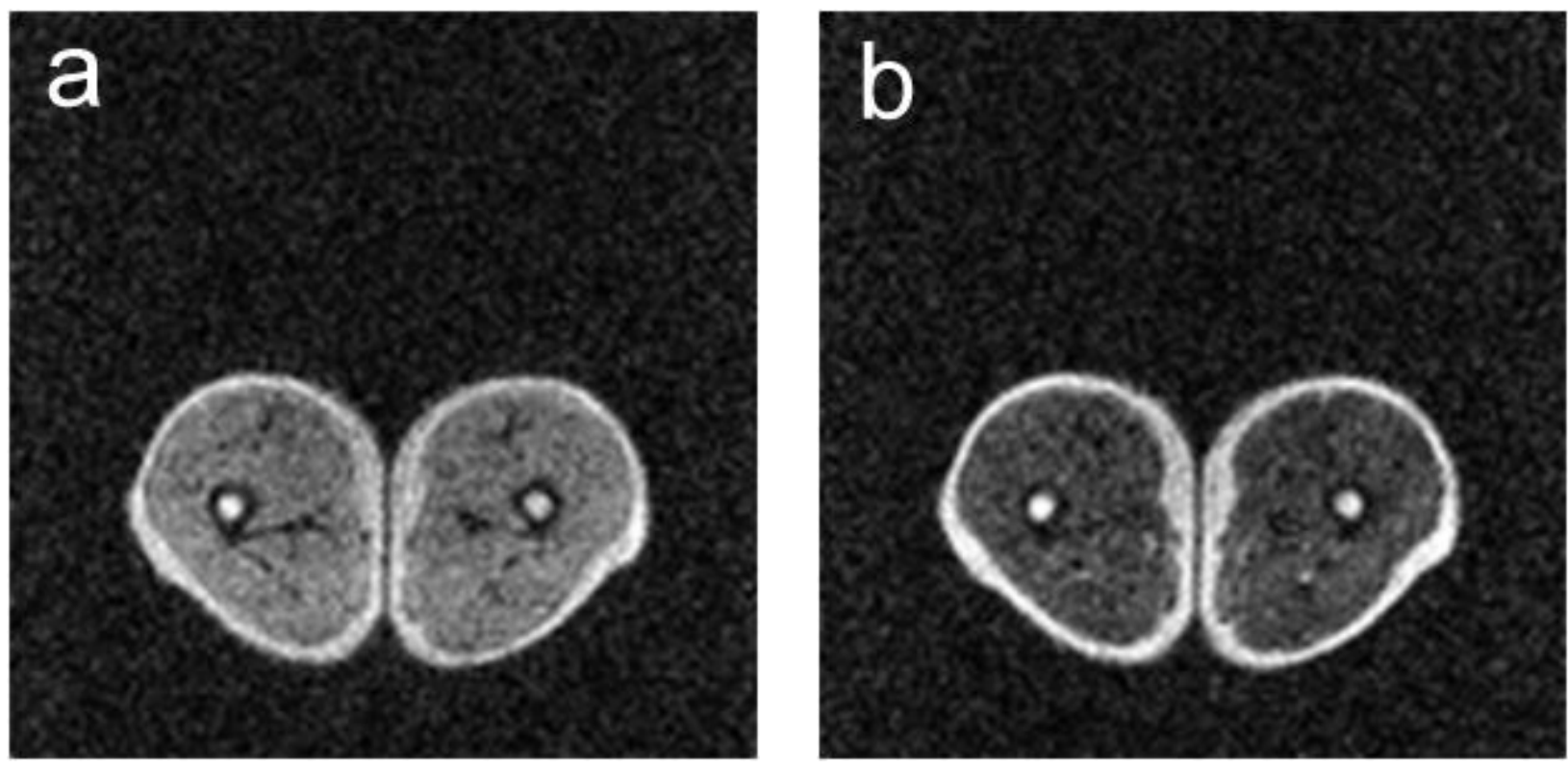

Figure 8: Inversion-recovery FFC-MRI images through the thighs of a normal volunteer. Detection field was 59 mT; slice thickness 15 mm; matrix 128×128; FOV $35 \times 35 \mathrm{~cm}$; TR $1500 \mathrm{~ms}$; TI $200 \mathrm{~ms}$; $\mathrm{NEX}=2$. Image a: $B_{0}{ }^{\mathrm{E}}=65 \mathrm{mT}$ (centre of quadrupole dip); Image $\mathrm{b}:{B_{0}}^{\mathrm{E}}=75 \mathrm{mT}$ (beyond quadrupole dip). Different image intensity in muscle is due to immobilised protein, giving significant difference in $T_{1}$. 

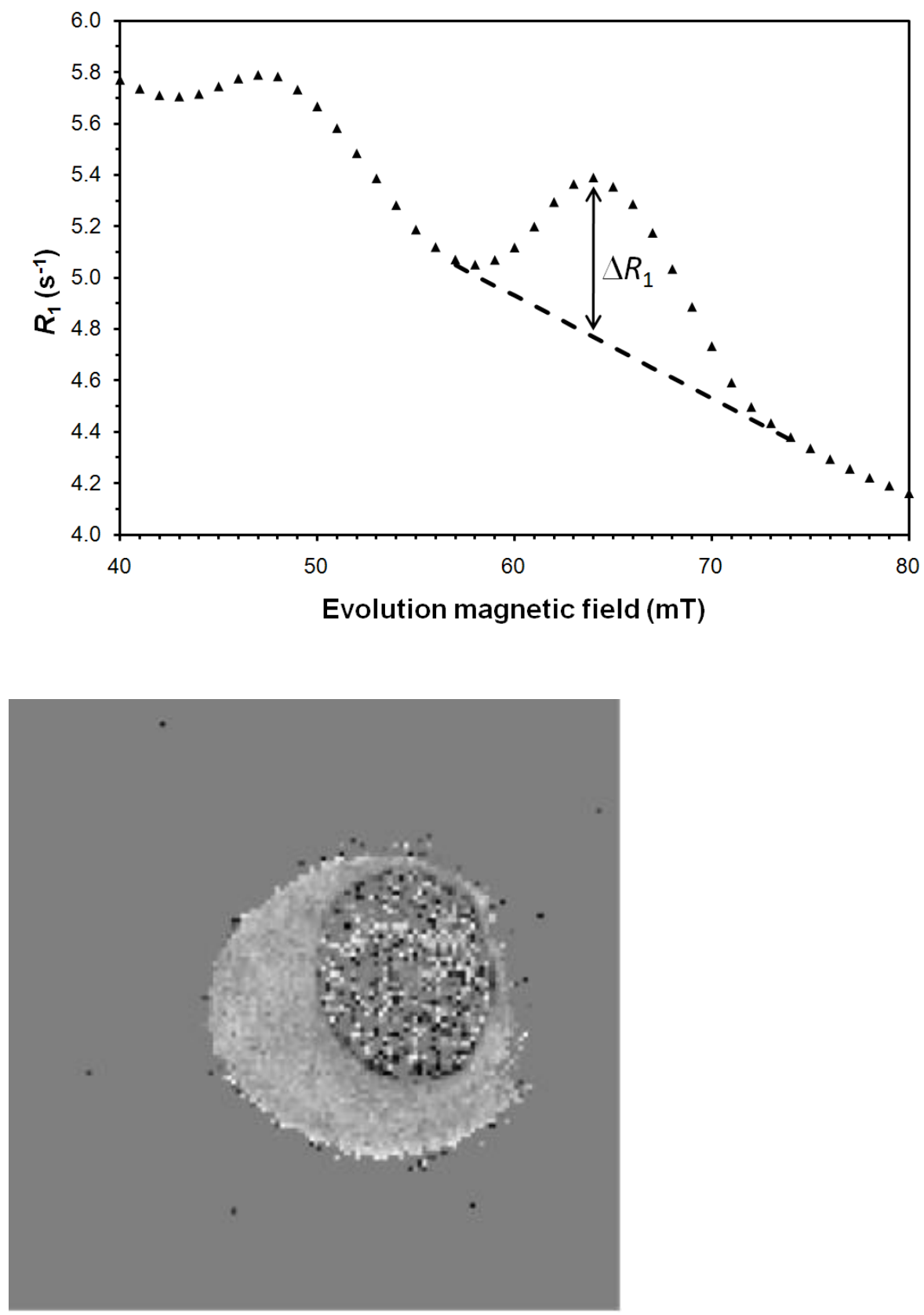

Figure 9: a $-R_{1}$ dispersion plot of heat-denatured hen's egg albumin, showing quadrupole peaks, obtained using $450 \mathrm{mT}$ FFC-MRI system. Also shown is the "height", $\Delta R_{1}$ of the $65 \mathrm{mT}$ quadrupole peak, in this case estimated as the difference between the measured $R_{1}$ and the relaxation rate in the absence of the quadrupole peak, estimated by linearly interpolation (dashed line). $\mathrm{b}$ - Calculated $\Delta R_{1}$ image of heat-treated hen's egg, obtained using interleaved inversion-recovery FFC-MRI on $450 \mathrm{mT}$ FFC-MRI system. Mid-grey background corresponds to $\Delta R_{1}=0$. Immobilised protein in albumin is evident from higher image intensity. 

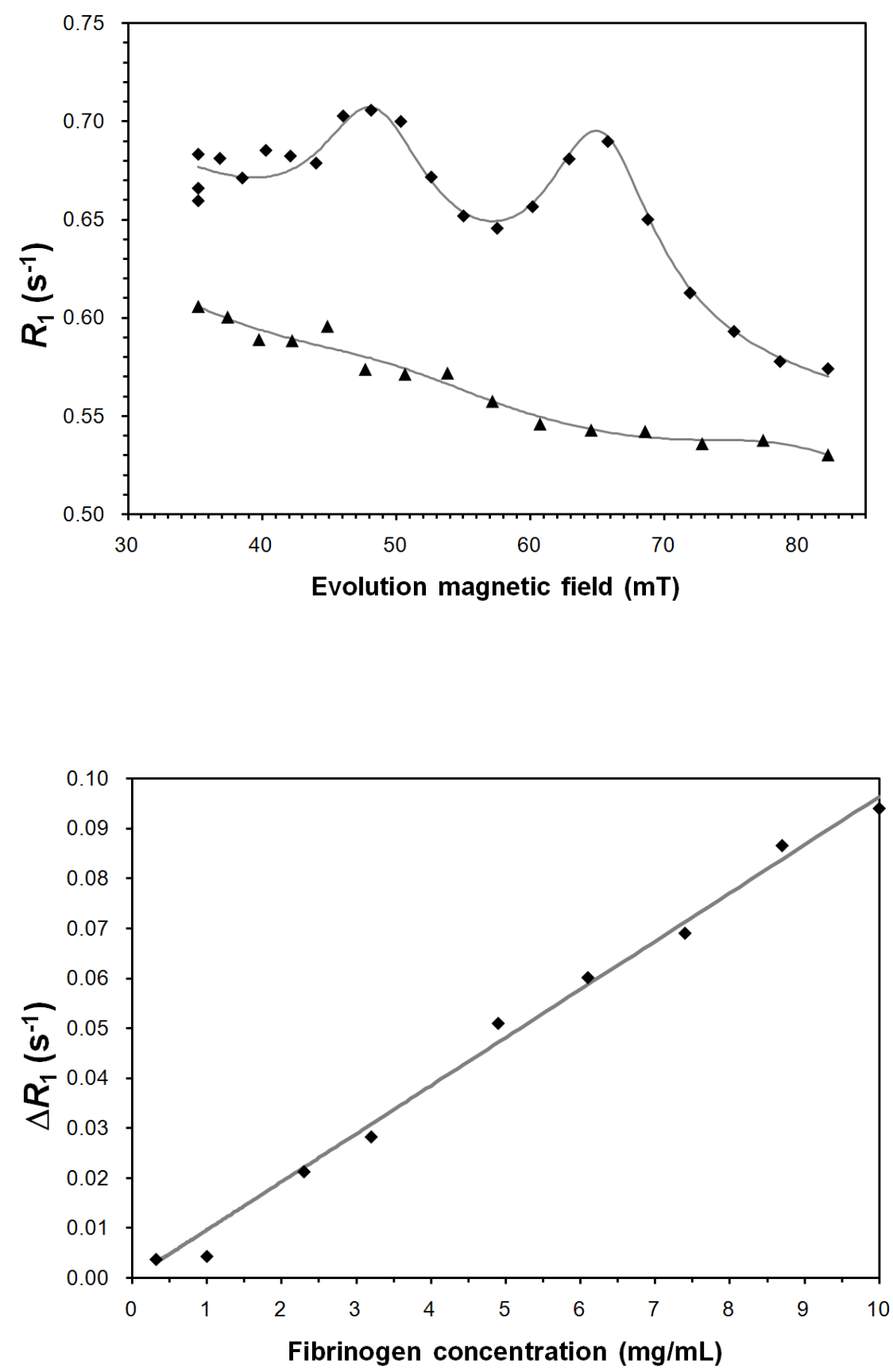

Figure 10: a - Dispersion curves of fibrinogen protein in aqueous solution (triangles) and cross-linked fibrin model clot (diamonds). Solid curves are fits using power-law background and Lorentzian peaks. Measurements made using commercial relaxometer (“SMARtracer", Stelar S.r.l., Italy). b - Plot of height of $65 \mathrm{mT}$ quadrupole dip $\left(\Delta R_{1}\right)$ versus concentration of fibrinogen in original solution, showing that $\Delta R_{1}$ is linearly dependent on concentration of cross-linked fibrin in the model clot. Linear fit (solid line) has $\mathrm{R}^{2}=0.99214$. 

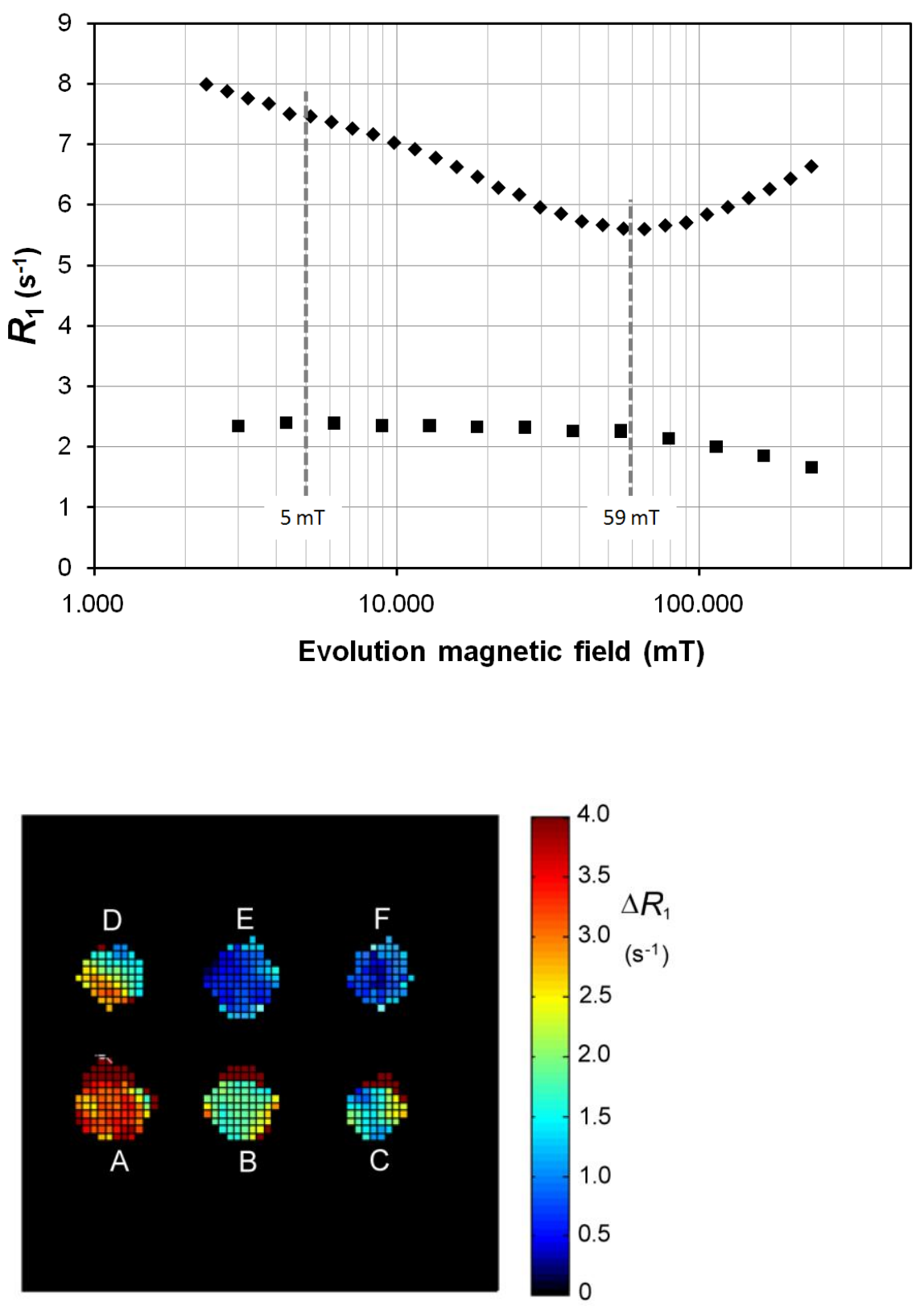

Figure 11: a - Measured dispersion plots (linear-log plot) for Mn(II) liposome suspension (average [Mn] $0.15 \mathrm{mM}$ ) (diamonds) and for $1 \mathrm{mM}$ aqueous solution of $\mathrm{CuSO}_{4}$ (squares). Vertical lines indicate evolution fields of $5 \mathrm{mT}$ and $59 \mathrm{mT}$ at which images were obtained. A significant difference in $R_{1}$ between these two fields can be seen for the liposome sample, but not for $\mathrm{CuSO}_{4} \cdot \mathrm{b}$ - Colourcoded image showing calculated difference between $R_{1}$ at $5 \mathrm{mT}$ and $59 \mathrm{mT}$. Samples A-E are Mn(II) liposome suspensions with $[\mathrm{Mn}]=$ A:- $0.15 \mathrm{mM}$; B:- $0.1 \mathrm{mM}$; C:- $0.08 \mathrm{mM}$; D:- $0.06 \mathrm{mM}$; E:- 0.04 $\mathrm{mM}$. Sample $\mathrm{F}$ is an aqueous solution of $1 \mathrm{mM} \mathrm{CuSO}_{4}$. 


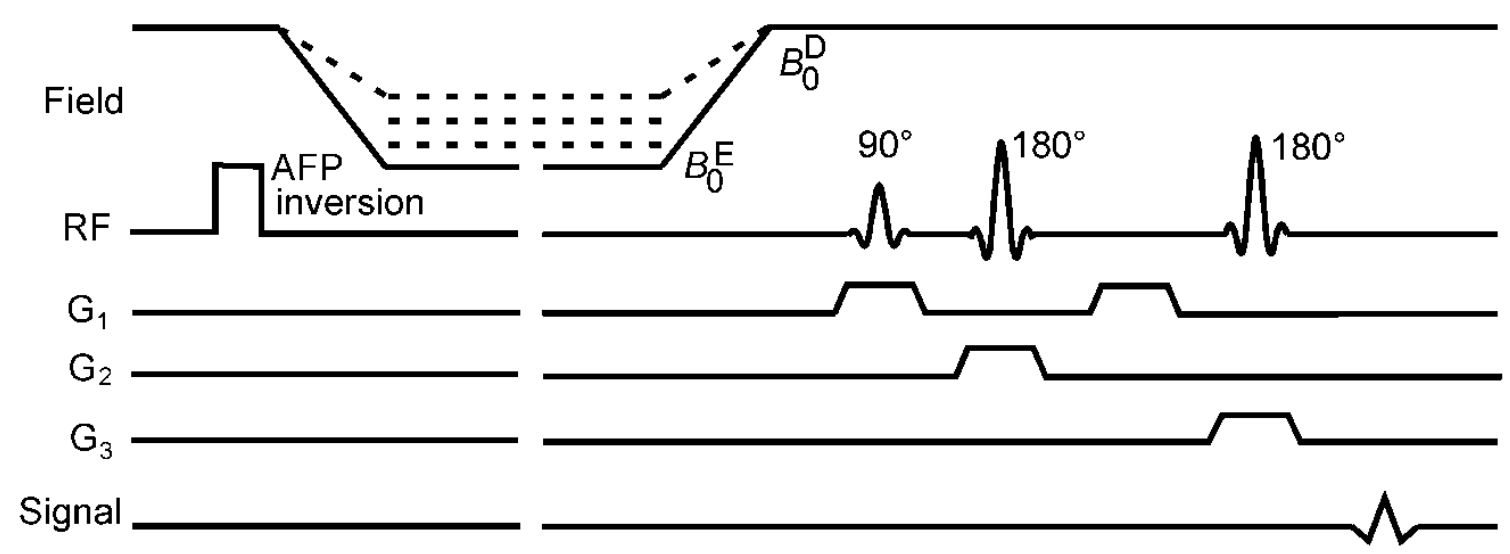

Figure 12: FFC relaxometry pulse sequence, using PRESS-like volume localisation (selective $90^{\circ}$ and two selective $180^{\circ}$ pulses, applied with mutually orthogonal gradients $G_{1}, G_{2}$ and $G_{3}$. 

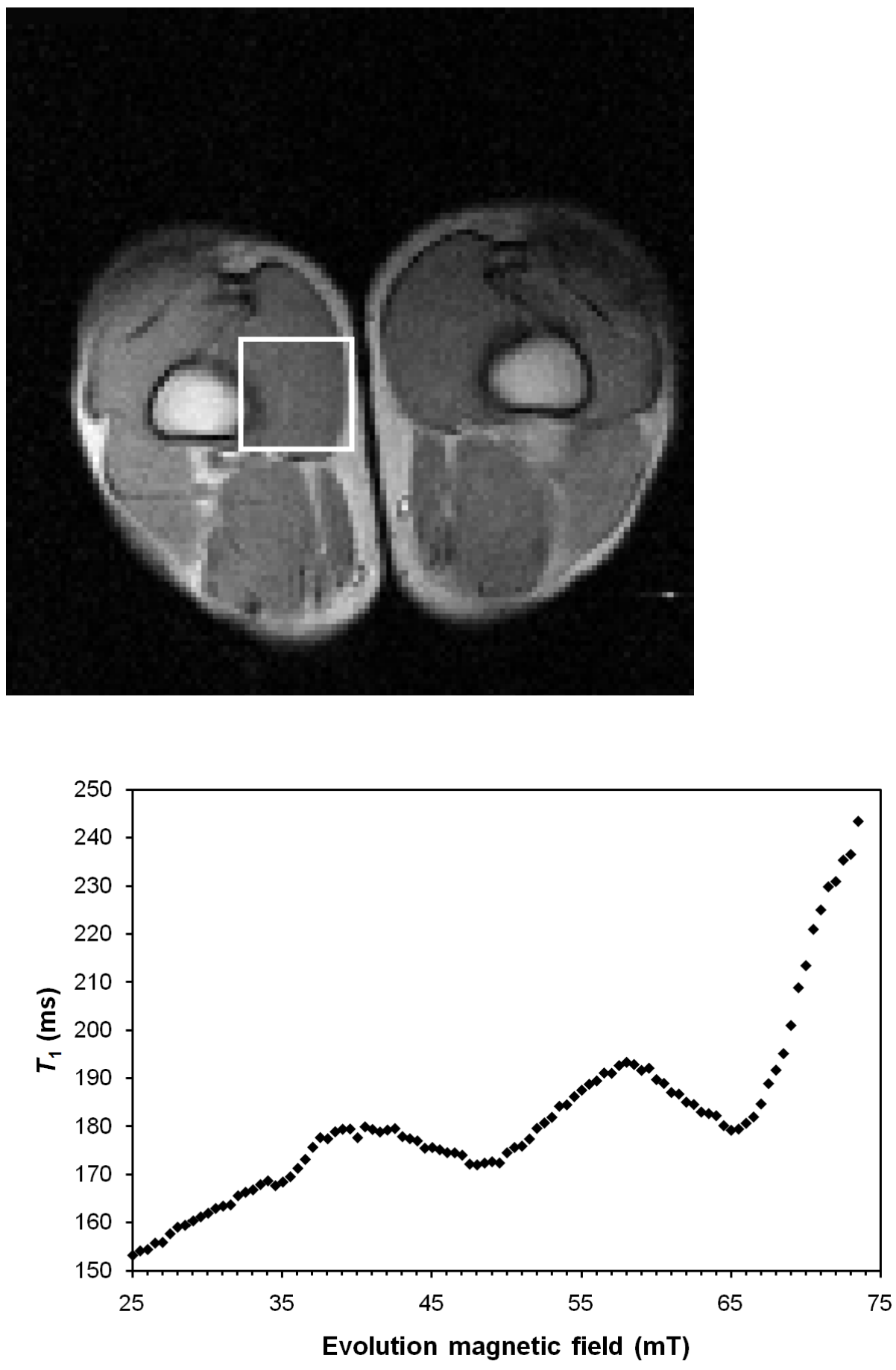

Figure 13: a - Gradient-echo image through thighs of a normal volunteer. White square shows region (mainly muscle) selected for subsequent volume-selected relaxometry. Image was obtained at $59 \mathrm{mT}$; matrix 128×128; FOV 25×25 cm; slice thickness $40 \mathrm{~mm}$; TR $300 \mathrm{~ms}$; NEX = 2. b - Volume-localised $T_{1}$ dispersion curve, measured from region shown on image. Selected volume is a cube of side $40 \mathrm{~mm}$. Plot has been filtered using a 5-point running average. Quadrupole dips at $49 \mathrm{mT}$ and $65 \mathrm{mT}$ are clearly evident. 